$4 \mid 2017$

Technologies numériques et diffusion de l'information pendant les périodes de conflits et de crise dans le monde

Discours de haine sur internet : entre la violence du cadrage biopolitique et le langage éthique du visage

Hate speech on the internet: between the violence of biopolitical framing and the ethical language of the face

Discurso de ódio na internet: entre a violência do enquadramento biopolítico e a linguagem ética do rosto

Frederico Vieira et Angela Cristina Salgueiro-Marques

\title{
OpenEdition
}

Édition électronique

URL : http://journals.openedition.org/ctd/691

DOI : $10.4000 /$ ctd.691

ISSN : 2491-1437

Éditeur

Chaire Unesco Pratiques émergentes en technologies et communication pour le développement

Référence électronique

Frederico Vieira et Angela Cristina Salgueiro-Marques, « Discours de haine sur internet : entre la violence du cadrage biopolitique et le langage éthique du visage ", Communication, technologies et développement [En ligne], 4 | 2017, mis en ligne le 04 juillet 2017, consulté le 29 mars 2021. URL: http://journals.openedition.org/ctd/691 ; DOl : https://doi.org/10.4000/ctd.691

Ce document a été généré automatiquement le 29 mars 2021.

Communication, technologies et développement 


\section{Discours de haine sur internet : entre la violence du cadrage biopolitique et le langage éthique $d u$ visage}

Hate speech on the internet: between the violence of biopolitical framing and the ethical language of the face

Discurso de ódio na internet: entre a violência do enquadramento biopolítico e a linguagem ética do rosto

Frederico Vieira et Angela Cristina Salgueiro-Marques

1 Dans le cadre des réflexions actuelles de certains auteurs concernant la conversation civique en ligne (Cramer Walsh, 2004; Kim et Kim, 2008; Gastil, 2008; Conover; Searing, 2005; Rojas, 2008; Mansbridge, 1999), les échanges jouent un rôle très important dans la création de cadres de référence partagés et utilisés par les individus dans l'objectif de comprendre les principales questions qui les concernent. Ils soutiennent que les conversations civiques leur donnent l'opportunité d'échanger des expériences de façon à mieux comprendre les raisons qui soutiennent les points de vue de leurs interlocuteurs (George, 2002).

D'après Kim et Kim, la conversation quotidienne «favorise des opportunités pour réfléchir à ses propres idées, réduire l'inconsistance cognitive en augmentant la qualité des opinions et des arguments individuels» (2008, p. 61). En se présentant comme un processus d'assemblage de faits, témoignages, informations et histoires, la conversation expose les individus à plusieurs idées et facilite la découverte et la compréhension des points communs et des divergences existantes entre eux (Black, 2008 ; Young, 1996).

3 En ce sens, Dahlgren (2000), Bohman (2004) et Miège (2006) soulignent que la médiation rendue possible par internet décentralise la sphère publique, car les espaces conversationnels de ce réseau abritent plusieurs publics au lieu d'une sphère publique unifiée et universelle. Ne nous y méprenons pas: les conversations peuvent autant 
aider que détruire les valeurs démocratiques et civiques. Certaines de nos interactions sont plus coopératives alors que d'autres sont plus conflictuelles et nous éloignent des autres au lieu de nous rapprocher. D'autres types de conversation peuvent avoir une fin en eux-mêmes ne conduisant pas à la coopération, à la confiance ou à l'engagement civique. Toutefois, ils sont aussi importants pour la convivialité et la sociabilité (Mutz, 2006 ; Rojas, 2008 ; Gastil, 2008). Parmi nos interactions communicatives, toutes ne sont pas orientées vers l'intercompréhension, la discussion de thèmes politiques ou la promotion d'objectifs démocratiques. Il est possible d'observer que, dans les espaces d'échanges en ligne, l'intensification du débat et l'exploration des points de discordance sont traversées par l'hostilité et par la non- acceptation de la différence.

4 Certains auteurs (Suraud, 2007; Dahlgren, 2005; Lev-On et Manin, 2006; Dahlberg, 2007 ; Janssen et Kies, 2005 ; Bohman, 2004) indiquent que les conflits discursifs qui donnent origine à des espaces publics en ligne sont habituellement marqués par un type d'incivilité lié à l'expression de cadres de dévalorisation, de cadrages interprétatifs méprisants et de déni de reconnaissance (dans certains cas il est possible de voir des éléments d'harcèlement moral et psychologique). L'incivilité peut à la fois empêcher les échanges argumentatifs (l'amortissement et l'attaque à la figure de l'interlocuteur, ce qui conduit à son outrage) ou bien les encourager dans des situations particulières, dans lesquelles l'antagonisme entre les différentes positions rendent sensibles les interlocuteurs à poursuivre et à mieux justifier leurs points de vue à la lumière de sa délégitimation, discrédit et questionnement (Anderson et al.,2014).

5 Les discours sociaux (juridique, médical, scientifique, religieux, entre autres) délimitent toujours des lieux de production du sens autant pour les individus que pour les institutions. En se référant constamment les uns aux autres, les discours construisent quelques cadres interprétatifs à travers lesquels se tisse une toile qui nous laisse voir les interstices, les distances et les vides qui caractérisent les relations entre les discours et les sujets. Dans ce jeu discursif, il est possible de discerner les relations de pouvoir allant de la soumission la plus tenace à l'hospitalité accueillante de l'Autrui. Les premières seraient au service du renforcement violent de la "vie nue » et, à l'autre extrême, les relations d'hospitalité rendent possible l'existence sociopolitique d'autres dimensions des processus subjectifs imbriqués dans les possibles de la communication parlée : la biopuissance de la singularité du visage.

6 L'objectif principal de cet article est de faire une brève analyse communicationnelle des tensions violentes créées par les mots blessants adressés aux "Nordestinos" du Brésil qui ont été publiés sur le réseau socionumérique du site web Tumblr, en soulignant les visages violés et thématisés par les énoncés produits et échangés entre les usagers. Comment considérer les discours haineux sur internet à partir d'une perspective éthique qui entremêle le cadrage interprétatif, la biopolitique et l'altérité du visage de l'autre?

7 Cette question est d'abord examinée d'un point de vue théorique qui prend en considération le rôle joué par les opérations de cadrage dans les échanges discursifs en ligne. Puis, à partir de données issues d'une analyse empirique, nous souhaitons mettre en exergue le caractère violent des posts adressés aux Nordestinos. Notre réflexion porte aussi sur la biopolitique et la façon dont les sujets exercent l'oppression les uns sur les autres à partir d'une introjection des modes contemporains de gouvernement des dominés. L'absence de reconnaissance issue des cadrages haineux amène à des traumatismes psychiques et sociaux, à des souffrances et à l'auto dévalorisation. En ce 
sens il y a une modalité particulière du gouvernement des corps collectifs : le cadrage biopolitique des populations en conflit (ou la biopolitique du cadrage collectif), c'est-àdire que l'ensemble de récits, d'arguments et des termes qui, utilisés de façon hostile par les participants des réseaux sociaux, soutiennent les modes de vie considérés comme dignes et reprochent véhémentement les modes de vie largement perçus et jugés comme méprisables.

8 Le cadrage biopolitique est ainsi une technique qui nous fait voir quelles sont les populations (groupes d'êtres qui partagent un espace défini politiquement, avec des traits biologiques particuliers, mais qui peuvent être configurés par l'usage des techniques et savoirs spécifiques) exemplaires, irréprochables et intouchables au détriment de celles dont la culture et le comportement sont pointés comme méprisables et indignes de reconnaissance.

\section{Le contexte sociopolitique brésilien et la haine aux « Nordestinos »}

On appelle "Nordestino" celui ou celle qui habite la région Nord-Est du Brésil (nommé Sertão), une région marquée par la pauvreté et le manque de ressources. Il est important de dire que cette façon de parler est chargée de préjugés et stéréotypes liés au passé esclavagiste de cette région. Il faut aussi remarquer que, tout au long du XXe siècle, les Nordestinos ont migré du Sertão vers les métropoles de la région Sud-Est à la recherche de meilleures conditions de vie et de travail et sont souvent devenus une partie de ces populations qui vivent dans les zones marginales (en devenant des squatters, en habitant les banlieues, les bidonvilles, entre autres).

10 Au Brésil, les taux de formalisation croissante du marché de travail depuis 2010 ne résolvent pas le problème du bas niveau salarial (Lautier, 2012). La "gestion de la pauvreté" (Cabanes et Georges, 2014) révèle les manières dont l'État stimule l'entrepreneuriat social, le commerce informel, les activités "culturelles » dans les banlieues, les politiques sociales dans le secteur de l'assistance et les formes de mécénat d'entreprise. En prenant en considération le fait que les politiques sociales obéissent à une chaîne capitaliste dont les donneurs d'ordre sont des multinationales, il est possible d'avoir comme résultat local de la mise en œuvre du Programme Bourse Famille un mélange entre droits et religion, reconnaissance et remerciement, justice et don.

11 Les politiques gouvernementales organisées à l'échelon national et les structures municipales qui les mettent en œuvre n'échappent pas à l'arrière-plan symbolique et culturel qui réaffirme constamment des jugements dévalorisants et méprisants envers les personnes pauvres (qui, dans ce cadre, ne sont pas en position d'acteurs sociaux, mais d'agents de dispositifs de pouvoir qui ne valorisent pas les capacités et n'encouragent pas l'empowerment).

12 À l'occasion de la réélection de Dilma Roussef ${ }^{1}$ en 2014 à la présidence de la République, les discours haineux sur Tumbrl à l'égard des Nordestinos ont augmenté drastiquement. La campagne présidentielle de 2014 a été marquée par le combat entre Dilma (Parti des Travailleurs) et Aécio Neves (Parti de la Social Démocratie Brésilienne - PSDB) et par une polarisation intense au deuxième tour. Dilma a remporté $51,64 \%$ des votes et Aécio Neves $48,36 \%$. La différence de votes a été de 3,4 millions, différence la 
plus faible depuis le retour à la démocratie. Dans tous les États du Nord-Est (régions plus pauvres du pays), la présidente réélue a remporté la majorité des votes, tandis qu'Aécio a été au sommet au Sud et au Centre-Ouest (régions plus riches du pays). Par contre, dans les régions du Sud-Est et Nord la dispute a été équilibrée ; dans les États de Rio de Janeiro et de Minas Gerais, par exemple, Dilma a rassemblé plus de votes, alors qu'à São Paulo et Espírito Santo, le sénateur a reçu la majorité des votes. Il faut souligner donc que Dilma était la candidate soutenue par les Nordestinos (la candidate des pauvres) tandis qu'Aécio était le candidat défendu par les Paulistas (le candidat des riches).

13 Suite à la victoire de Dilma Rousseff (PT) contre Aécio Neves (PSDB) pour le poste de Président de la République, en octobre 2014, deux sites web : "Esses Nordestinos..." et «Esses Paulistas... » ont été publiées sur la plateforme Tumblr. Selon ses créateurs, les deux pages ont été créées afin de dénoncer les expressions de haine circulant sur l'internet et incitant des conflits entre Nordestinos et Paulistas. Nous soutenons que ce conflit donne à voir, à travers le dispositif de cadrage interprétatif (Butler, 2015), une forme spécifique de biopolitique qui met en place un mécanisme de valorisation et reconnaissance des populations placées au Sud-Est brésilien au détriment de la dévalorisation des modes de vie, des corps et des valeurs culturelles des populations $d u$ Nord- Est. Cette biopolitique du cadrage collectif des populations régionales d'un pays construit discursivement et aussi justifie la construction de frontières et de formes de jugement moral qui définissent ceux qui doivent être respectés et ceux qu'il faut " gifler, brûler, menacer, exterminer ", selon les mots utilisés par les individus qui ont échangé des posts sur les deux sites web analysés ici.

Selon Fassin (2009) et Lazzarato (2002), la biopolitique est principalement liée aux expériences concrètes dans lesquelles les individus sont traités de façon juste ou injuste, selon quels principes et au nom de quelle morale, conduisant fréquemment à des inégalités et au manque de reconnaissance. La vie et le vivant sont les enjeux des nouvelles luttes politiques; pourtant il ne faut pas les voir uniquement par les cadrages étatiques et institutionnels, mais principalement par les récits et les échanges qui exposent des blessures, des violences et des souffrances vécues par des populations incitées à ne pas voir dans l'autre un égal, voire même un être humain.

\section{Considérations méthodologiques}

D'après Dahlberg (2007) et Stromer-Galley (2002), l'internet peut offrir des espaces de conversation politique où les dominés peuvent contester les perspectives dominantes et apporter de nouvelles perspectives au débat en le complexifiant. Les conditions de politisation se trouvent justement dans la capacité des agents de tisser des arguments capables de relier leurs récits individuels et les faits concrets qui témoignent de leur oppression. Les espaces de conversation en ligne, créés autour d'une revendication ou d'une contestation partagée par un groupe minoritaire ont pourtant tendance à réunir un nombre restreint de personnes ayant le même statut social, le même niveau d'études et les mêmes intérêts (Lev-On et Manin, 2006; Miège, 2006). Cela peut réduire ces espaces à des arènes repliées sur elles-mêmes, cantonnés au mieux à un rôle d'espace d'expression à la visibilité minime.

16 Si nous prenons en considération le fait que les communautés et groupes dans les réseaux sociaux numériques partagent un lexique singulier de connaissances, 
expériences et énoncés, les échanges restreints deviennent significatifs pour définir les référents d'un groupe, et donc leurs identités. La manifestation de certains aspects identitaires dans les conversations en ligne ne tient pas uniquement aux valeurs et à l'affirmation du cadre interprétatif individuel de chaque participant, elle est aussi liée à la représentation des groupes et des communautés en relation.

De façon générale, les dynamiques discursives instaurées dans les espaces numériques de discussion mettent en place des procédures de publicisation qui se révèlent gênantes, car elles exposent les participants à des "questions sensibles». Warren (2006) définit ces questions comme celles qui exposent les fragilités et les intimités des individus au regard des autres. Discuter à propos d'une question sensible peut alors révéler des expériences d'exclusion et des relations de déni de reconnaissance qui renvoient les interlocuteurs aux positions dévalorisées par la société.

Ce qui nous intéresse à propos de ces relations c'est la présence d'une violence symbolique aigue, car la distinction "nous »/ " eux » est généralement faite de façon agressive, antagoniste, voire belliqueuse. Notre objectif général est de voir comment des opérations de cadrage mises en place par différents groupes en dispute peuvent donner lieu à l'expression de l'intolérance et de la haine, et aussi contribuer au renforcement de valeurs et croyances méprisantes et qui rendent impossible le dialogue dans les espaces de communication du réseau numérique. Ainsi, les opérations de cadrage sont aussi des opérations de pouvoir.

Étant donné cet objectif nous avons fait une sélection d'environ 63 posts issus de deux blogs spécifiques: http://essesnordestinos-blog.tumblr.com et http:// essespaulistas.tumblr.com. La récolte des données a été réalisée le 15 juin 2015, dans ces deux blogs. Le blog «Esses Nordestinos » avait 31 archives de prints des posts qui vocalisaient des discours de haine contre les personnes du Nord-Est, suivis des commentaires de l'auteur du blog et des notes des lecteurs, dont les dates de publication variaient entre les 5 et 7 octobre 2014. À son tour le blog "Esses paulistas » avait 32 archives de prints de posts qui vocalisaient des discours de haine contre les personnes du Nord-Est, aussi suivis des commentaires de l'auteur du blog et des notes des lecteurs, dont les dates de publication variaient entre les 6 et 28 octobre 2014. Ainsi la collecte a considéré l'univers de 63 archives pour l'analyse publiés au long du mois de juin 2015. La méthodologie qualitative a porté sur les aspects textuels et visuels des messages, sans oublier l'architecture discursive du Tumblr (possibilités d'échanges, mécanismes de contrôle, éléments du langage et éléments d'identification identitaire). L'axe principal des analyses a pris appui sur l'association entre ces aspects de façon à cerner l'expressivité du message et spécifiquement le sentiment réciproque de haine vers l'autre dans les conversations en ligne. Nous avons observé celui « dont les sujets parlent» (soit du Nord-Est, soit Paulistano) et la façon d'adresser le message (« à qui la parole s'adresse »). Les catégories de postages, à leur tour, ont été formulées à partir du contenu des messages, rendant possible de regrouper les posts qui avaient en commun la thématique génératrice des expressions de haine. Ensuite, nous avons sélectionné les posts considérés comme plus représentatifs de ces regroupements pour développer la réflexion basée sur les concepts de cadrage, visage et biopolitique.

Ainsi nous avons essayé de garantir une l'analyse qui ne soit pas limitée à la description du phénomène, mais tournée vers une critique de ce qui dépasse, ce qui est vu et dit dans les unités d'analyse (posts), en privilégiant les dédoublements politiques et leurs effets sur le processus de présentation, vocalisation et dissémination de la haine par/ 
sur autrui. En un mot, les concepts ont été convoqués dans l'analyse dans la mesure où la l'énonciation du texte et de l'image d'un post les évoquaient et pas l'inverse.

\title{
Celui-là : les cadrages haineux qui définissent l'Autre
}

\author{
"Comment gagner le vote d'un "Nordestino" ? Il faut juste lui donner à manger \\ avec une carafe d'eau. Allez travailler. » Luiza Moreira ${ }^{2}$. \\ «Le truc c'était de donner le statut d'état indépendant au Nord-Est du Brésil! \\ Hahaha (rire) Bande d'abrutis. » Rafael Castro. \\ « $70 \%$ de votes pour Dilma dans le Nord-Est du Brésil. Médecins du Nord-Est, \\ pratiquez l'holocauste chez vous! On doit changer cette réalité !! A Auteur non \\ identifié.
}

Ces trois déclarations, extraites de publications qui circulent sur le réseau numérique, sont un petit échantillon d'un large éventail de discours verbaux et visuels dont la violence contre les autres - identifiés ici comme «les gens du Nord-Est du Brésil », l'Autre généralisé et subalterne - varie de l'ironie grossière à l'offense brutale, de la blague méprisante à l'attentat odieux dirigés vers ceux qui diffèrent "du même", de celui conçu par le parleur. Les gens parlent sur les réseaux et pointent le différent comme un ennemi. D'après eux il faut éliminer les formes de vie considérées comme indignes et qui ne tolèrent pas la même vision politique et sociale, fondée sur des rapports de domination historiquement construits.

Parmi les différents circuits de communication en ligne créés par des individus qui se révèlent à travers leurs messages, la conversation et le bavardage virtuel agressif sont mis en évidence, due à l'absence, dans une large mesure, de la réglementation étatique ${ }^{3}$. Le manteau de l'« anonymat " fourni par le réseau numérique permet souvent à l'individu de parler librement et de façon illimitée en utilisant profils réels ou faux, en s'adressant à toutes les personnes ou à des groupes d'amis, en discutant sur tous les sujets possibles.

Le texte de présentation du site "Esses Nordestinos... » réjette les opinions dénoncées en encourageant les lecteurs à faire de même: "Voulez-vous apporter votre contribution? Envoyer des copies des manifestations xénophobes à ce tumblr aide à exposer le problème et à susciter la discussion"[sic]. En outre, la page fournit un lien (une entrée) pour ceux qui veulent porter plainte: " Si vous voulez faire un pas en avant et faire répondre les auteurs de ces publications, envisagez de porter plainte au Ministère Public Fédéral sur leur site internet. »[sic]. Le site "Esses Paulistas... », Créé en octobre de la même année, présente des justifications et des stratégies similaires à la page dédiée à la violence symbolique contre les Nordestinos. La réaction commence par son titre : «Les préjugés envers les Paulistas : ça se fait? » ATTENTION : L'AUTEUR DE CE BLOG EXCLUT TOUTE SORTE DE PRÉJUDICE SOIT CONTRE LES BRÉSILIENS DU NORD, DU SUD, DE SAO PAULO, ETC... "[sic $]^{4}$

À première vue nous voyons dans la page "Esses Paulistas..." une répétition du positionnement agressif trouvé dans le site dédié à "Esses Nordestinos... 》 Cela contribue à une évidente polarisation politique et identitaire soutenue par des discours de haine contre les subjectivités apparemment dites subalternes (placées au Nord-Est) et d'autres connues comme dominantes (placées au Sud-Est / Sao Paulo). Voici quelques exemples : "Le paulista ne vote pas. Il fait caca dans l'urne électorale", José de Abreu ; "Savez-vous ce qui manque à ces Paulistes ? D'être giflés!, Boulettes de Dépression ; "MEC, JE VAIS BRÛLER CES CONNARDS DE PAULISTAS", Flawless. Dans ce scénario, le 
trafic de la communication bipolaire suscite le conflit symbolique violent autour du champ politique, motivé par le résultat des élections. Les affrontements sont présents et incitent à des discours politiques de haine, qui sont renforcés dans l'espace public générant des pratiques sociales violentes, mais également des pratiques de résistance. Ces violences ne se limitent pas au domaine verbal, mais apparaissent concrètement dans les rues.

Ne serait-elle pas valable, dans une certaine mesure, l'exposition de ces discours, mise en lumière par les posts, pour expliquer les préjugés et permettre l'inversion du sens de l'agression en les différenciant et en rompant avec la norme hostile? Une fois visibles, les posts font émerger dans l'espace public la force d'une caractéristique enracinée dans la culture politique du pays.

Nous nous demandons si cette émergence provoque un pouvoir émancipateur de guérison, au- delà de la promotion de la maladie sociale. Dans quelle mesure est-il possible d'identifier dans le choc discursif entre les sites "Esses Nordestinos... » et « Esses Paulistas... » une opportunité d'affirmation des altérités réifiées, en stimulant la haine totalisante envers le différent? Par la suite, nous verrons, dans un examen plus approfondi des posts, la présence de différents cadres interprétatifs qui nous amène au dispositif d'énonciation révélateur du niveau de privilège des déclarations, tandis que les paroles de subalternes restent encore réduites au silence dans les deux blogs.

Une opération de cadrage mise en place par les médias et par les énoncés numériques est une opération politique de pouvoir comme l'a remarqué Judith Butler: cadrer quelqu'un signifie aussi définir les manières de le juger et d'agir envers cette personne. Selon Butler (2015, p. 14), les cadres (ou cadrages interprétatifs) « ne déterminent pas de manière unilatérale les conditions de l'apparaître (apparence au sens d'une apparition performative sur la scène publique) des sujets et des événements, leur finalité est néanmoins de délimiter la sphère d'apparition. " Ainsi, les cadres nous aideraient à distinguer les vies dont nous pouvons saisir, apprécier et valoriser celles qui ne méritent pas d'être considérées. Les termes, les catégories, les conventions et les règles générales qui opèrent dans des dispositifs de cadrage modèlent et transforment, par exemple, un être vivant en un sujet reconnaissable à travers une forme spécifique d'appréhension et connaissance. Selon Bulter (2015, p. 20) «le problème n'est pas simplement de savoir comment inclure davantage de gens dans les normes existantes, mais de considérer la manière dont ces normes distribuent la reconnaissance sur un mode distinct. »

Les prémisses, les jugements, les désaccords, les valeurs, les prédispositions, etc., qui permettent aux acteurs sociaux de reconnaître et de saisir des faits et des perceptions tout en créant des histoires ou des rapports cohérents, révèlent comment le cadrage est configuré comme une idée organisatrice centrale dans un processus de dévoilement et de construction discursive d'un problème politique et des sujets politiques précaires.

Il faut souligner que les cadres interprétatifs issus des médias et des espaces de communication comme les plateformes numériques expriment un conflit symbolique dans lequel chacune des parties impliquées s'engage différemment. Il s'agit d'un processus conflictuel et antagoniste de description et construction narrative d'un problème public en partant de différents "récits ", " personnages » et "arguments ", qui sont fortement influencés par les logiques opératoires et les mécanismes discursifs des médias. En outre, la concurrence entre des cadres différents ne garantit pas que les parties opposées vont s'affronter en conditions de parité, ou bien que le public soit 
exposé également et simultanément à des cadres alternatifs et à des répertoires tout aussi convaincants.

Il a été possible d'identifier certains répertoires communs entre les posts autour d'idées clés. Dans le site "Esse Nordestinos..." les interlocuteurs évoquent les associations sémantiques suivantes : (1) misère/pauvreté liée aux conditions de vie et de travail au Nord-Est du Brésil. Ces conditions sont perçues comme du retard économique et comme forme d'immobilisme social, ce qui peut être confirmé par exemple par ces expressions : "Allez travailler », "Vagabonds », « Les pauvres sont de la merde. »

La sauvagerie des jugements inscrit le "peuple» du Nord-Est brésilien dans le bas clergé du royaume des animaux, en les comparant avec l'âne, un "peuple sans culture ». La race abjecte trace la distinction "nous-eux", en révélant la face biopolitique la plus perverse : l'extermination des formes indésirables de vie : «cette race qui a de quoi vivre avec mon argent"; "provoquer l'holocauste au Nord-Est brésilien"; "Putains de racailles". Les déclarations caractérisées par des éléments scatologiques, malédictions et imprécations sont des agressions capables d'effacer la potentialité de la vie en faveur d'un mécanisme biopolitique de contrôle.

Par contre, dans le site "Esse Paulistas...." nous avons des "variations sur le même thème", avec quelques différences. Le seul élément vraiment nouveau est l'utilisation de la sécheresse urbaine comme appât pour mettre sur la table le thème de la catastrophe écologique et sociale vécue actuellement par les Paulistas. Cette catastrophe est mobilisée comme instrument de punition et de torture d'autrui, en libérant les sujets de leur responsabilité envers le phénomène environnemental. Dans l'ensemble, sont évoqués des associations sémantiques dans ce blog: (1) la précarité urbaine est liée à des conditions de vie indignes dans la métropole : "SÃO PAULO EST TERRE DE GENS DÉGUEULASSE, TANT PIS L'EAU ! ! ? Félicitations Paulistes, vous méritez vraiment le métro bondé... ", Vinicius Barbieri. Les Paulistas méritent, selon les interlocuteurs du blog, "l'eau du Tietê (une rivière polluée) pour boire", " Les accro au crack", "Le trafic de merde", "L'éducation périmée » et « le pire que ce gouvernement crétin et corrompu a à nous offrir..." ; (2) La bestialité est liée particulièrement à l'homophobie: "Pédés", "sales couchons" ; (3) la race abjecte renforce la distinction «nous-eux »: « race malheureuse, pleine de préjugés, meurtriers depuis l'époque des pionniers dans la découverte du Brésil » ou « berceau des homo ».

Un post d'auteur non identifié fait des efforts pour catégoriser les préjugés contre les Paulistas, en leur donnant des variations régionales: "Les insultes dirigées aux Paulistas dans le Sud-Est sont liées à leur sang mitigé (...) dire que les Paulistas sont un petit peu « sales » est commun à certains égards, là-bas. » [sic].

Cette publication interdiscursive nous offre un regard critique de la région Sud vers les Paulistas, étant donné que le Sud s'affirme comme terre des gens de "sang pur" en opposition au "caboclo sujinho"5 (petit, méprisable et sale comme le Paulista). Il convient de souligner la forte concentration de colonies d'origine européenne dans le Sud brésilien en particulier les Germaniques et les groupes néonazis. Historiquement, le mouvement séparatiste gaucho renforce également l'identité locale/régionale, en superposant monolithiquement l'appartenance de cette région au pays et en déconsidérant les altérités multiples qui l'aggravent. 


\section{La nudité biopolitique et les ruptures inspirées par Lévinas}

Depuis sa naissance la démocratie occidentale a été définie à partir du binôme exclusion-inclusion, entre Polis et Oikos. La condition de la citoyenneté à Athènes, par exemple, a été réservée au groupe privilégié constitué des hommes grecs considérés en tant qu'orateurs dans l'espace public de l'agora et auxquels de nombreux êtres apolitiques comme les femmes, les enfants, les esclaves et les métèques ont été soumis.

La Zoé s'est constituée comme une vie inférieure vis-à-vis de la «bonne vie » liée à l'expérience philosophique et politique. Elle était considérée comme obscure et destinée aux soins du monde domestique, du maintien du corps et moyens d'assurer la survie de l'espèce. Avec l'avènement de la république à Rome, installée sur un système juridique dont l'influence atteint nos jours, le concept de Vita a permis de réunir sous la même juridiction la vie digne et la vie indigne.

37 La figure juridique de l'homo sacer (Agamben 2002, p. 56) apparait alors pour désigner un homme sacré, pas de la même manière que le sens commun le comprend aujourd'hui, mais comme l'homme qui ne pouvait pas participer à la res publica. Son existence était dénuée de droits et distincte des citoyens romains ; l'homo sacer était susceptible d'être tué sans que sa mort soit vue comme un crime.

Des siècles plus tard, après l'apogée et le déclin du christianisme dans les domaines de l'État - au cours duquel on a témoigné à nouveau d'une nouvelle polarisation entre la chair et l'esprit, le corps et la faculté de la raison - le début du XXe siècle a accompagné, avec Heidegger, la consolidation de la pensée philosophique qui, en même temps, avait restitué la prévalence de l'Être sur la condition humaine ordinaire. L'Être de Heidegger diffère de celui de l'entité, se libère de son aspect de « chose » et a son origine dans la relation établie avec le temps, inexorable.

Heidegger a fondé, à partir de la tradition phénoménologique allemande, une ontologie qui cherchait à comprendre la finitude de l'homme, son être-là, l'être en attente de la mort qui est abandonné à son propre sort face à l'horizon de l'Être. Bien que son questionnement sur l'existence ait rompu partiellement avec la cosmovision grecque, la perspective ontologique du langage chez Heidegger a vidé l'être (l'existant) de son protagonisme concret, en l'abstrayant dans sa propre solitude finie. Une telle vision renforcerait, dans son extrême, l'anonymat d'un être déjà dévalué, réduit à la condition équivalente d'homo sacer.

40 Fait révélateur, l'ontologie de Heidegger de Sein und Zeit a rendu possible, à partir des engagements politiques pris par le philosophe lui-même avec le national-socialisme allemand, une tournure philosophie qui justifie finalement la montée du Reich allemand, l'état d'exception par excellence du XXe siècle. Sur cette scène politique, les camps de concentration nazis et le registre sombre de cadavres empilés à côté des fours crématoires ont constitué l'une des plus grandes horreurs de la violente nudité biopolitique dans la contemporanéité. Les corps supprimés, les visages effacés et les voix des altérités subalternes réduites au silence ont trouvé dans l'anonymat la demeure finale d'une vie digne qui différait de l'identité allemande, de la race aryenne.

41 Après Heidegger, Michel Foucault a utilisé le terme biopolitique en dénonçant les techniques de contrôle collectif des corps et les procédures de totalisation et en valorisant le rôle des pratiques discursives dans ce processus et des dispositifs par 
lesquels elles opèrent. La théorie de la biopolitique chez Foucault est apparue en 1976, dans le premier volume de l'Histoire de la Sexualité. Foucault (1984) nous explique la transition du pouvoir souverain (qui fait mourir et laisse vivre) au biopouvoir (qui fait vivre et laisse mourir) : il s'agit de deux régimes, deux logiques, deux conceptions de la mort, de la vie et du corps. Dans ce nouveau régime, le pouvoir est destiné à produire des forces censées stimuler la vie, la créativité et la productivité qui ne restent pas libres d'un contrôle et des limites.

Ainsi, le biopouvoir s'occupe du gouvernement des corps à travers une rationalisation de l'art de gouverner qui a une incidence sur les modes de vie une fois qu'il cherche la normalisation des personnes à travers des technologies politiques (Fassin, 2009). Le «faire vivre » caractéristique du biopouvoir peut assumer deux formes principales : la discipline (domination et optimisation des forces du corps individuel/ corps-machine), et la biopolitique, qui opère non sur les personnes, mais sur la population (corpsspécimen). La biopolitique envisage la vie à partir de la perspective du comportement, dans des termes de technologies qui normalisent les populations. Il y a ainsi une différence entre biopouvoir (l'art de gouverner par le régime disciplinaire imposé aux corps individuels) et biopolitique (production et gouvernement de nouvelles formes de vie) : la biopolitique mise en place au XIXe siècle n'est pas une politique de la vie, mais une politique de la population, considérée comme une communauté d'êtres vivants.

La combinaison des pratiques disciplinaires et des biopolitiques a pour objectif l'insertion contrôlée des sujets dans la machine capitalistique de production en augmentant les chances d'une adaptation aux besoins du marché et aussi d'une mise en forme ou bien du cadrage normalisant des corps et des subjectivités (Lazzarato, 2002 ; Hardt ; Negri, 2000).

Foucault interroge le biopouvoir en quête de ce qui dans la vie lui résiste par la création des formes de subjectivation qui échappent aux biopouvoirs. Le rapport éthique à soi et aux autres est, d'après Foucault, la source de l'émancipation et de la création transformatrice impliquées dans tous les rapports de pouvoir. En ce sens la pensée de Foucault diverge de celle d'Agamben pour qui la biopolitique détruit inévitablement la puissance de la vie en la soumettant à la régulation juridique et l'État d'exception. Nous ne pouvons pas nier qu'Agamben a raison puisque les agencements institutionnels, les interventions militaires, les reconfigurations de l'espace urbain en vue des mégaévénements par exemple sont toujours entamés au nom de la défense des droits et la préservation de la vie. Ce constat renforce la nature paradoxale de la biopolitique chez Agamben: le maintien de la qualité de vie d'une collectivité implique la destruction de la vie des autres. Néanmoins, l'approche d'Agamben ne nous donne pas beaucoup de pistes sur les possibilités de résistance à la biopolitique. C'est alors que le travail de Hardt et Negri (2000) mérite attention, car ils ont pris la trace de Foucault pour donner nouveau sens au concept de biopolitique: contre la reproduction de modes de vie et des consciences préapprouvées ils détectent la biopuissance des relations de coopération et hospitalité, des agencements créatifs et des liens politiques et esthétiques (Pelbart, 2003, 2002).

\section{Biopolitique des corps encadrés et des modes de vivre biopuissants}

Étant donné la difficulté de mettre en question les cadrages biopolitiques des populations dans un pays, comment détecter la voix de l'autre, son cri, ses demandes, 
son cri de douleur, mais aussi d'émancipation aux paramètres consensuels, aux captures du capital ? Une réponse possible est l'éthique du visage tel que développé par Lévinas et repris par Butler $(2011,2015)$. Le concept du visage nous permet de définir la biopolitique non plus comme pouvoir sur la vie (la vie ici perçue comme survie, comme un fait naturel, biologique, zoè), mais comme puissance de la vie (la vie comme source de créativité et réinvention), des formes de subjectivation qui débordent du cadre défini par les stratégies étatiques, institutionnelles et médiatiques. Affronter le visage de l'autre requiert de rompre avec des opérations biopolitiques de cadrage et de catégorisation des populations.

Le concept de visage, développé par Lévinas tout au long de son travail depuis Totalité et Infini (1961), vise à répondre à des questions éthiques importantes qui configurent, $a$ priori, notre propre humanité, dans laquelle l'Autrui nous précède et nous présente des altérités devant lesquelles il faut faire face, car on ne peut pas échapper à l'appel éthique de l'Autre. Le visage chez Lévinas doit être compris en dehors de la manifestation concrète sur des "faces » humaines au sens strict; le visage lévinassien possède un caractère indiciel dans les visages humains, montrant l'infini de l'altérité. Selon le philosophe, le visage ne vocalise pas par des mots, mais par quelque chose qui les précède; le visage est incommensurable et ne se laisse pas thématiser. "La manifestation du visage est déjà discours. Celui qui se manifeste porte secours à luimême, selon le mot de Platon. Il défait à tout instant la forme qu'il offre par sa manifestation. » (Lévinas, 1980, p. 53)

La notion de visage nous permet de reconnaître des traces capturées par le regard qui formulent une sorte d'interpellation éthique chez nous; nous sommes convoqués à donner une réponse à l'accueil de l'Autrui, totalement différent de moi. Le visage nous adresse un appel à la responsabilité, non seulement sur ce que nous faisons, mais également devant le mal qui peut nous atteindre. Face au visage, nous sommes affectés dans nos projets et l'autodéfense est toujours traversée par l'impératif de l'énoncé : "Tu ne tueras pas", déclaré par le visage. Le visage apparaît ainsi chez Lévinas dans l'infinité de manifestations concrètes de l'Autrui, comme ce qui est différent du Soi et qui devrait être considéré a priori dans le domaine de la politique. Le visage acquiert ses contours dans la réponse à cet appel éthique provenant d'Autrui : « répondre au visage, doit être une compréhension de la précarité de l'Autre ». (Butler, 2011, p. 19)

Nous croyons que la vue d'ensemble de tous les cadres et les paroles (les dits) construit un paysage discursif totalisant qui colonise les visages. En méprisant le différent, les visages révèlent et approfondissent les préjugés et les stéréotypes connus et présents dans le discours des médias, des fondamentalistes religieux homophobes, des conservateurs xénophobes alignés avec les philosophies de la droite radicale. Selon Butler (2011, p. 28 et 29) la « vie précaire $»^{6}$ devient un substrat pour la représentation imagétique de tout ce qui est vu comme improbablement humain.

49 Les polarisations apparentes comme le "Nous/Eux", "Sud/Nord", "États Parasites/États Producteurs", PT/PSDB (les partis politiques les plus influents au Brésil) deviennent homogènes par des pratiques discursives de la haine qui utilisent des répertoires communs construits par différents mots qui ne font que masquer le même sens totalitaire.

50 Ce que l'on voit, c'est la suppression de la parole et des perspectives dissonantes à la haine, ainsi que l'effacement des visages qui ne sont pas cadrés par la violence biopolitique au sein des pratiques langagières. Les interlocuteurs regardent de manière 
fixe le visage du subalterne et réifient leur altérité : voici le pauvre indifférencié qui reçoit la Bolsa Familia ; prend le métro bondé ; vu parfois comme stupide, parfois comme pédé; le vagabond qui travaille pour avoir du pain et de l'eau, même si cette dernière est prise dans la rivière Tietê (égout); le subalterne dans sa nudité, celui classé comme racaille, auparavant homo sacer, à présent de la race abjecte, la plèbe, le reste. La violence symbolique des discours qui se nourrissent de façon autopoietique permet aux subjectivités et leurs visages d'être absorbés à l'intérieur du dit en devenant anonymes. Par ailleurs, le langage chez Lévinas a une biopotence : elle ne garde pas les paroles, mais offre le vide à l'autrui pour qu'il remplisse de sens les paroles (Ribeiro, 2015).

51 La simultanéité des physionomies circule à bonne distance parmi les identités et les altérités, mais invalide le tiers, en renferment son visage dans lesdits, en tant qu'un être-là (Dasein) anonyme encapsulé dans la relation Nord-Est - São Paulo. Il y a plusieurs Nord-Ests dans ses visages infinis; comme le sont aussi les paroles qui décrivent l'urbanité complexe au sein du cosmopolitisme paulista. Nous nous demandons si parmi les subtilités discursives de Tumblr, le tiers a le droit de prendre la parole et, ainsi, d'exprimer la clameur qu'entremêle voix et visage chez Lévinas.

En ce sens, la présence cryptée du visage d'autrui dans les manifestations constantes de la haine dans les messages de «Esses Nordestinos... » et «Esses Paulistas..... » doit diriger ces messages au-delà du dit et périmètre, dans une certaine mesure, défaire leur dire par le passage d'un tiers dans la représentation.

L'extrait choisi considère la condition de finitude de l'existence humaine ; la brièveté et la corporéité incontournable à l'être. L'idée d'égalité est évoquée, par exemple, par un post d'une femme nordestina, que donne la réponse suivante à des injures haineuses: "Quand nous mourons nous allons tous au même trou ».

La mort - et notre condition humaine fragile devant elle - donne curieusement du visage à l'indignation et à l'outrage qui atteint le visage de la nordestina ; la figure de la mort lance un tiers dans la tension centre/périphérie. Celle-ci est imposée par le discours de la haine, qui de la même façon met en égalité la condition du nordestino et du sulista, à un certain moment, même dans un temps incertain.

À ce stage, le discours de la haine ne fonctionne pas, il ouvre une fente, une fissure pour le passage du visage lévinassien. De manière subtile, la figure de la mort défait le dit, elle forme le «nous » et redonne un autre sens à la haine manifestée par les agresseurs. La biopuissance est l'agencement qui permet de recréer constamment les modes d'apparition, d'énonciation et d'interlocution pour faire face à la haine et rendre possible l'écoute du visage.

\section{BIBLIOGRAPHIE}

Ashley Anderson, Dominique Brossard, Dietram Scheufele ; Michael Xenos ; Peter Ladwig, "The Nasty Effect : Online Incivility and Risk Perceptions of Emerging Technologies", Journal of Computer-Mediated Communication, v.19, 2014, pp. 373-387. 
Azi Lev-On ; Bernard Manin, « internet : la main invisible de la délibération », Esprit, mai, 2006, pp. 195-212.

Bernard Miège, «Pratiques informationnelles et culturelles et usages des Tics : differentiation voire individualisation?». Text présenté dans l'École d'été du GdR Tics, 2006.

Davy Janssen ; Rapahel Kies, « Online forums and deliberative democracy : hypotheses, variables and methodologies ", Acta Política, v.40, 2005, pp. 317-335.

Diana Mutz, Hearing the Other Side : Deliberative versus Participatory Democracy, New York, Cambridge University Press, 2006.

Didier Fassin, “Another politics of life is possible", Theory, culture \& society, v.26, n.5, 2009, pp. 4460.

Emmanuel Lévinas, De outro modo que ser ou para lá da essência, Trad. : José Luiz Pérez e Lavínia Leal Pereira, Lisboa, Centro de Filosofia da Universidade de Lisboa, 2011.

Emmanuel Lévinas, Ética e infinito, Lisboa, Edições 70, 2007.

Emmanuel Lévinas, Totalidade e Infinito, Trad. José Pinto Ribeiro, Lisboa, Edições 70, 1980.

Éric George, « Dynamiques d'échanges publics sur internet », In Francis Jauréguiberry ; Serge Proulx (dirs), Internet, nouvel espace citoyen ?, Paris, L'Harmattan, 2002, pp. 50-79.

Giorgio Agamben, Meios sem fim : notas sobre a política, Trad. Davi Pessoa, Belo Horizonte, Autêntica Editora, 2015.

Giorgio Agamben, Homo Sacer : o poder soberano e a vida nua I, Belo Horizonte, UFMG, 2002.

Hernando Rojas, » Strategyversus Understanding : howorientationstowardpoliticalconversation influence political engagement », Communication Research, v.35, n.4, 2008, pp. 452-480.

Iris Young, " Communication and the Other : Beyond Deliberative Democracy », In Seyla Benhabib (dir.), Democracy and Difference. Contesting the Boundaries of the Political, Princeton, Princeton University Press, 1996, pp. 120-135.

James Bohman, « Expanding dialogue : the internet, the public sphere and prospects for transnational democracy », Sociological Review, 2004, pp. 131-155.

Jane Mansbridge, » Everyday Talk in Deliberative System », In Stephen Macedo(dir.), Deliberative Politics : essays on democracy and disagreement, Oxford, Oxford University Press, 1999, pp. 211- 239.

Jennifer Stromer-Galley, « New voices in the public sphere : a comparative analysis of interpersonal and online political talk », The public, v.9, n.2, 2002,pp. 23-42.

John Gastil, Political Communication and Deliberation, London, Sage, 2008.

Joohan Kim ; Eun Joo Kim, « Theorizing dialogic deliberation : everyday political talk as communicative action and dialogue », Communication Theory, v.18, 2008, pp. 51-70.

Judith Butler, "Vida precária”, Contemporânea - Revista de Sociologia da UFSCar, SãoCarlos, Departamento e Programa de Pós-Graduação em Sociologia da UFSCar, 2011, n.1, pp. 13-33.

Judith Butler, Quadros de guerra : quando a vida é passível de luto ?, Rio de Janeiro, Civilização Brasileira, 2015.

Katherine Cramer Walsh., Talking about Politics : Informal Groups and Social Identity in American Life, Chicago, University of Chicago Press, 2004.

Laura Black, « Deliberation, Storytelling, and Dialogic moments », Communication Theory, v.18, 2008, pp. 93-116. 
Lincoln Dahlberg, « Rethinking the fragmentation of the cyberpublic: from consensus to contestation », New Media and Society, v. 9, n.5, 2007, pp. 827-847.

Luciane Ribeiro, A subjetividade e o outro : ética da responsabilidade em Emmanuel Levinas, São Paulo, Ideias e Letras, 2015.

Maurizio Lazzarato, « Du biopouvoir à la biopolitique », Multitudes, n.9, 2002, pp. 45-57. MarieGabrielle Suraud, «Communication ou délibération : les échanges dans la société civile », Hermès, v.47, 2007, pp. 177-184.

Mark Warren, « What should and should not be said : deliberating sensitive issues », Journal of Social Philosophy, v.37, n.2, 2006, pp. 163-181.

Michael Hardt ; Antonio Negri, “La production biopolitique », Multitudes, n.1, 2000, pp. 16-28.

Michel Foucault, História da Sexualidade I : vontade de saber, Trad. de Pedro Tamen, Rio de Janeiro, Graal, 1984.

Pamela Conover ; Donald Searing, «Studying 'Everyday Political Talk' in the Deliberative System », Acta Politica, v. 40, 2005, pp. 269-283.

Peter Dahlgren, «L'espace public et l'internet : structure, espace et communication », Réseaux, n. 100, 2000, pp. 159-186.

Peter Dahlgren, « The internet, Public Spheres, and Political Communication : dispersion and deliberation », Political Communication, n.22, 2005, pp. 147-162.

Peter Pál Pelbart, Vida Capital : ensaios de biopolítica, São Paulo, Iluminuras, 2003.

Peter Pál Pelbart, "Pouvoir sur la vie, puissance de la vie », Multitudes, n.9, 2002, pp. 25-35.

Teresa Sales, "Raízes da desigualdade social na cultura política brasileira", Revista Brasileira da Ciências Sociais, v.9, n.25, São Paulo, 1994, pp. 24-43.

\section{Sites internet consultés}

https://eleicoes.uol.com.br/2014/noticias/2014/10/26/dilma-cresce-na-reta-final-e-reeleita-eemplaca-quarto-mandato-do-pt.htm, consulté le 27/6/15, à $11 \mathrm{~h} 38$.

http://g1.globo.com/tecnologia/noticia/2014/10/pagina-compila-ofensas-paulistas-em-redessociais-apos-eleicao.html, consulté le 30/6/15, à 16 h08.

\section{NOTES}

1. La précarité et la pauvreté au Brésil ont diminué pendant les gouvernements Lula et Dilma Roussef (Parti des Travailleurs - PT), mais ce résultat ne rend pas compte de la persistance de nombreuses inégalités et asymétries de pouvoir entre les citoyens. Cabanes et Georges (2014) montrent que durant les années Lula (2003-2010) l'augmentation continue du salaire minimum a été accompagnée par celle des niveaux d'éducation, mais aussi par la mise en place de politiques sociales comme le Programme Bourse Famille (Bolsa Família, crée en 2003). Cependant, alors que la pauvreté a diminué, les inégalités sociales persistent, voire se creusent avec le travail précaire, les mauvaises conditions de vie et la fausse perspective d'acquisition de droits. La Bourse Famille par exemple n'est pas un droit acquis durablement, inscrit dans la Constitution. 
2. Pour des raisons d'éthique, les nom et prénom des auteurs des posts ont été modifiés.

3. Le Secrétariat aux Droits de l'Homme a créé en 2014 un groupe de travail spécialisé dans la cartographie des crimes contre les droits de l'homme sur les réseaux sociaux. Les messages surveillés par SaferNet ont mis en évidence une augmentation entre $300 \%$ et $600 \%$ de ce type de violation entre 2014 et 2014. La loi brésilienne est précaire et mérite une révision au sujet de la cybercriminalité. Selon le gouvernement, dans huit ans, SaferNet Brasil a reçu et traité 3417208 plaintes anonymes impliquant 527000 sites web. Les demandes ont été enregistrées par la population grâce à la création de hotlines qui intègrent le Centre National de Plaintes contre les Crimes Cybernétiques.

4. Il vaut noter que les commentaires du responsable du blog «Esses nordestinos..." ne s'éloignent pas des publications des internautes. Même ceux qui dénoncent le mépris répètent le mode agressif dans leurs déclarations : Comment faut-il savoir si votre ami est un enculé ? Attendez sa réaction aux résultats des élections. »; " Le vrai con on le reconnaît par des opinions comme celle-ci. » ; « Mon pote, je suis désolé, mais le con ici c'est l'autre. » [Sic]

5. Voir: http://movimentogauchoindependente.blogspot.com.br (Movimento Gaúcho Independente) et http://www.pampalivre.info/faq.htm (República do Pampa).

6. Selon Butler (2015), il devrait y avoir reconnais- sance de la précarité comme condition partagée de la vie humaine. "La précarité implique la vie sociale, c'est-à-dire le fait que la vie de quelqu'un est toujours en quelque sorte aux mains d'autrui. Elle implique que l'on est exposé à la fois à ceux que l'on connaît et à ceux que l'on ne connaît pas ; une dépendance par rapport à des gens que l'on connaît, que l'on connaît à peine ou que l'on ne connaît pas du tout » (2015, p. 31).

\section{RÉSUMÉS}

Cet article propose une réflexion à propos de la violence de l'affrontement entre les identités totalisantes et les altérités subalternes exprimées dans les posts (images et textualités) qui circulent sur le réseau numérique. Nous utilisons la perspective biopolitique (Foucault) pour analyser les pratiques discursives révélées dans les sites web sur Tumblr : « Esses nordestinos... » et « Esses Paulistas... ». Ceux-ci ont été créés après la réélection de Dilma Rousseff à la Présidence de la République en octobre 2014 et révèlent comment les populations du Nord-Est et du Sud-Est brésilien sont dirigées vers un conflit dont l'objet de dispute est aussi l'élément qui renforce les formes de contrôle et d'homogénéisation des groupes. Nous considérons dans les analyses les oppositions entre identités et altérités, selon la notion de cadrage interprétatif (Butler), la signification éthique du «Visage » (Lévinas) et la biopuissance (Hardt et Negri, Lazzarato, Pelbart).

This paper is a reflection about the violence of the conflict between totalizing identity and subaltern alterities that appear on posts (images and textualities) that are circulating on web social spaces of public exchange. We use the biopolitics perspective (Foucault) to analyze the discursive practices revealed in the Tumblr pages "Esses nordestinos"... and "Esses Paulistas...", created after the Dilma Rousseff's re-election for president of the republic, in october 2014. The online conversations showed how populations of Nord east and South east are compelled to get into a conflict that reinforces the means of controlling and the homogenisation of groups. Our analysis presents the oppositions between identity and alterities from the concepts of 
interpretative framing (Butler), ethical meaning of Face (Lévinas), and biopuissance (Hardt and Hegri, Lazzarato, Pelbart).

Neste artigo refletimos sobre a violência do embate entre identidades totalizantes e alteridades subalternas manifestas em posts (imagens e textualidades) que são produzidos em conversações políticas via web. Utilizamos a perspectiva biopolítica (Foucault) para analisar as práticas discursivas reveladas nas páginas "Esses nordestinos..." e "Esses Paulistas..." do Tumblr, criadas após a reeleição de Dilma Rousseff para a presidência da república, em outubro de 2014 e revelam como as populações do nordeste e do sudeste são levadas a um conflito no qual a disputa reforça formas de contrôle e de homogeneização de grupos. Na análise, consideramos as oposições entre identidades e alteridades a partir dos conceitos de enquadramento interpretativo (Butler), de significação ética do Rosto (E. Lévinas) e biopotência (Hardt e Negri, Lazzarato, Pelbart).

\section{INDEX}

Mots-clés : biopolitique, discours, visage, altérité, Tumblr

Keywords : biopolitics, speech, face, alterity, Tumblr

Palavras-chave : biopolítica, discurso, rosto, alteridade, Tumblr

\section{AUTEURS}

\section{FREDERICO VIEIRA}

Université fédérale de Minas Gerais

\section{ANGELA CRISTINA SALGUEIRO-MARQUES}

Université fédérale de Minas Gerais 